\title{
Do fragmentation functions in factorization theorems correctly treat non-perturbative effects?
}

\section{John Collins*}

104 Davey Lab, Penn State University, University Park PA 16802, USA

E-mail: jcc8@psu.edu

\begin{abstract}
Current all-orders proofs of factorization of hard processes are made by extracting the leading power behavior of Feynman graphs, i.e., by extracting asymptotics strictly order-by-order in perturbation theory. The resulting parton densities and fragmentation functions include nonperturbative effects. I show how there are missing elements in the proofs; these are related to and exemplified by string and cluster models of hadronization. The proofs rely on large rapidity differences between different parts of graphs for the process; but in reality large rapidity gaps are filled in.
\end{abstract}

QCD Evolution 2016

May 30-June 03, 2016

National Institute for Subatomic Physics (Nikhef), Amsterdam

\footnotetext{
* Speaker.
} 


\section{Introduction}

Factorization theorems (of both the collinear and the transverse-momentum-dependent (TMD) kinds) are intended to be applicable in full QCD. They apply to a number of important reactions to within an error suppressed by a power of a large scale. As well as perturbatively calculable components (notably hard-scattering factors, evolution kernels), the factorization properties include factors like parton densities and fragmentation functions that are intended to incorporate the relevant non-perturbative information.

However, the actual published proofs are normally made by a systematic analysis of the leading power in each order of perturbation theory. Therefore, since we claim that factorization properties do incorporate genuine first-principles predictions of QCD, we must ask how well we know that factorization is justified in full QCD, and not merely in order-by-order perturbation theory. This issue has been recognized for a long time, e.g., [1].

As we will see, there are many elements in an order-by-order derivation of factorization that do appear to have a validity beyond pure perturbation theory. The same applies to the parton-model intuition that motivated the factorization approach.

In this paper, I will show explicitly that, in contrast, there are certain parts of the derivation that clash with what we know of hadronization in the final state in QCD in hard collisions. These are the phenomena that are approximated in Monte-Carlo event generators under the heading of string or cluster hadronization [1]. The primary issue is that existing perturbative proofs of factorization need large rapidity differences between the different momentum classes considered, but hadronization fills in the rapidity gaps, as is known experimentally. The filling in of rapidity gaps can even be modeled within perturbation theory when the order of important Feynman graphs increases logarithmically with energy.

These results indicate that some physically important phenomena are not properly treated within the normal perturbatively-based derivations of factorization. This does not necessarily imply that factorization actually fails. But it does imply that our current understanding is incomplete.

\section{Factorization and the importance of non-perturbative components}

\subsection{Basics of factorization}

Hard-scattering factorization applies to many kinds of cross section with a large scale $Q$ (e.g., deeply inelastic scattering (DIS)), and has a typical form of a hard scattering convoluted with parton densities (pdfs) and/or fragmentation functions (ffs):

$$
\sigma=\text { hard scattering } \otimes \text { pdfs and/or ffs }+ \text { power-suppressed. }
$$

Pdfs and ffs have scale dependence that is governed by evolution equations, e.g., that of DGLAP. The predictive power given to QCD by factorization properties arises from the following:

- The hard scattering, the DGLAP kernels, etc can be usefully calculated by low-order perturbative calculations in powers of a small effective coupling $\alpha_{s}$. 
- In contrast, pdfs and ffs cannot be computed perturbatively. But they can be measured from a limited set of data over a limited range of $Q$, as can the scale parameter $\Lambda_{\mathrm{QCD}}$ for the evolution of $\alpha_{s}$.

- Then, after evolution is allowed for, the universality of pdfs, ffs, etc enables factorization to give predictions for many other processes at all high enough $Q$. Similarly it also gives predictions for cross sections for the processes used to measure the pdfs etc, but at different $Q$ values than those used for their measurement.

\subsection{Non-perturbative reasoning in coordinate-space motivates factorization/parton ideas}

The motivation that led to factorization theorems in QCD was the parton model. In the case of DIS, $e+p \rightarrow e^{\prime}+X$, the parton model assumptions are illustrated in Fig. 1, in the overall centerof-mass frame. There is a fast-moving incoming Lorentz-contracted, time-dilated proton (or other hadron). The incoming electron scatters through a wide-angle hard scattering from a single constituent of the hadron, over a small distance scale of order $1 / Q$. This is much smaller than the time scale $Q / m^{2}$ for valence processes inside the hadron, where $m$ is a typical hadronic scale. Within the pure parton model, it is assumed that the hard scattering is a lowest-order graph only (with exchange of a virtual photon), without higher-order strong-interaction corrections. Then the cross section is schematically $\mathrm{d} \sigma=$ hard sc. $\otimes$ pdf.

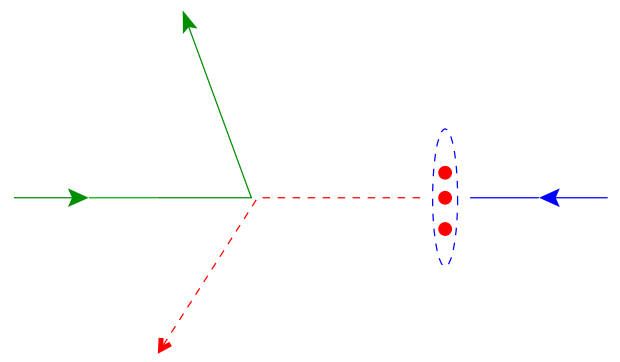

Figure 1: DIS in parton model, with a proton coming from the right and an electron from the left. The hard scattering is off one constituent (red, dashed) of the proton.

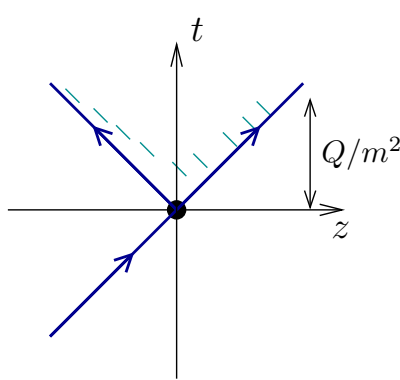

Figure 2: Space-time view corresponding to Fig. 1 in the Breit frame.

The space-time structure is illustrated in Fig. 2 in the Breit frame, i.e., where the exchanged virtual photon and the proton are in the $(t, z)$ plane. The processes that lead to the hadrons in the final state are dominantly roughly on a space-like hyperbola at an invariant distance of order $1 / \mathrm{m}$ from the hard scattering. The hadronization region is indicated by the series of short diagonal green lines.

Hadronization happens much too late to affect the fully inclusive cross section; it just rearranges the final state from a struck quark and the proton remnant to something else. Thus the parton-model cross section is correctly given by the product of hard scattering and parton density, without any allowance for the hadronization processes. Notice how the validity of this argument relies both on the use of unitarity for the final-state interactions and on a coordinate-space picture that arises from the highly relativistic kinematics. 


\subsection{Fragmentation in $e^{+}+e^{-} \rightarrow h_{1}+h_{2}+X$ at high $Q$}

To examine the hadronization process in more detail, we consider $e^{+} e^{-} \rightarrow$ hadrons at centerof-mass energy $Q$. We use a parton-model-like approximation, illustrated in Fig. 3, with its lowest order hard-scattering. A quark-antiquark pair is created over a short distance scale, of order $1 / Q$, which is followed by their propagation and hadronization, with the space-time structure indicated in Fig. 4. The two-particle-inclusive (2PI) cross section with hadrons in opposite hemispheres is sensitive to the hadronization process, and will bring in the relation to ffs.

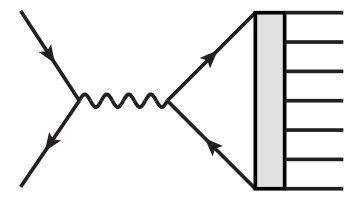

Figure 3: Amplitude for $e^{+} e^{-} \rightarrow$ hadrons to lowest order in hard scattering, with an indication of hadronization.

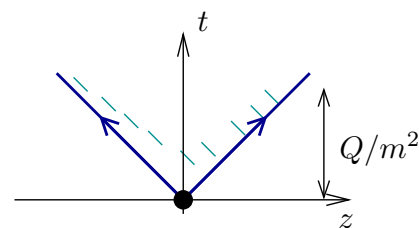

Figure 4: Space-time view corresponding to Fig. 3.

A well-motivated model for hadronization is the string model $[2,3,4,5]$. Between the outgoing quark and antiquark is generated a gluonic color flux tube. Quark-antiquark pairs are generated in the flux tube, and these rearrange themselves into color-singlet hadrons, mostly pions; these fill in the rapidity gap between the quark and antiquark with an approximately uniform distribution in rapidity. The diagram in Fig. 3 is rather misleading, since it ignores the fact that the flux tube is created by gluon emission quite early compared with the $Q / \mathrm{m}^{2}$ time-scale for the generation of the fastest hadrons. The central hadrons are generated relatively early, on a time-scale $1 / \mathrm{m}$. The space-time structure, Fig. 4, follows from [2, 3, 4, 5].

The fastest oppositely moving hadrons are generated at space-like separation. This and stringlike fragmentation implies independent fragmentation on the two sides, and so for hadrons in opposite hemispheres, the approximations used so far can reasonably be expected to give a factorized 2PI cross section with ffs, of the schematic form

$$
\frac{\mathrm{d} \sigma}{\mathrm{d} z_{1} \mathrm{~d} z_{2}}=\text { hard sc. } \otimes \mathrm{ff}_{1}\left(z_{1}\right) \otimes \mathrm{ff}_{2}\left(z_{2}\right)+\text { power suppressed. }
$$

However, because the rapidity gap is filled in, there are Feynman-graph-like structures connecting the two opposite jets.

\section{Standard proofs of factorization}

For a systematic treatment of factorization and its derivation, see [6], where also references to previous literature can be found.

\subsection{Overview}

Given some suitable process with a large scale $Q$, the overall aims are to extract the leadingpower large- $Q$ asymptote of each individual graph for process, and then to obtain a factorized form 
after a sum over graphs. (Sometimes a non-leading power is also treated.) This is carried out, in typical proofs, along the following lines:

1. The regions that give the leading large- $Q$ behavior are determined.

2. For each region, appropriate valid approximations are applied. The aims are that

- They should be suitable for obtaining a factorized cross section.

- They should allow the use of the Ward identities, as noted below, to combine graphs.

3. For each term for each region, subtractions are applied to avoid double counting, etc.

4. Ward identities are applied to extract gluons connecting subgraphs for different classes of momenta (i.e., soft-to-collinear, collinear-to-hard).

5. Any relevant final-state unitarity cancellations are proved.

6. The result is a factorized form after a sum over graphs.

Evolution equations (DGLAP, etc) are derived similarly.

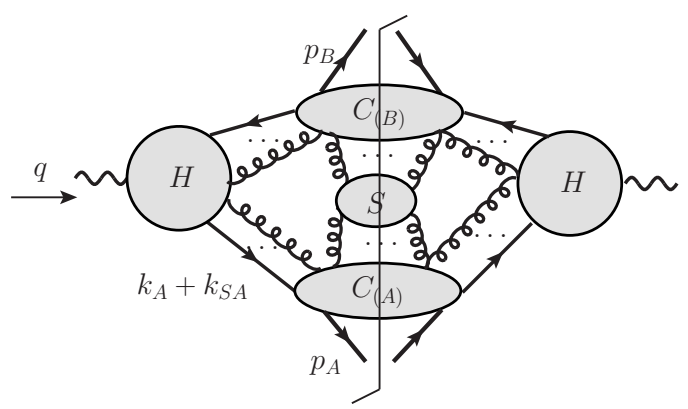

(a)

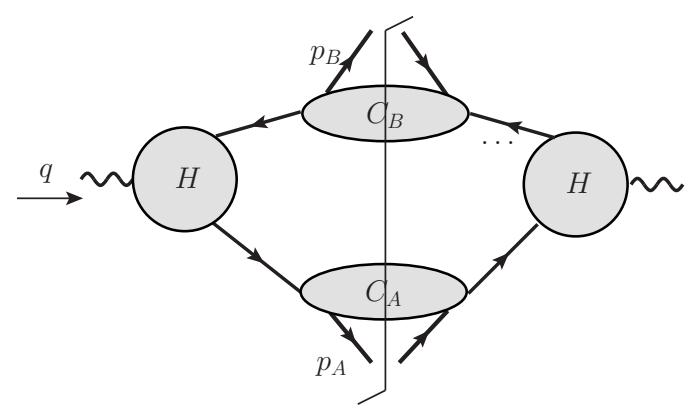

(b)

Figure 5: Leading regions for $e^{+}+e^{-} \rightarrow h_{1}+h_{2}+X$ : (a) in full QCD; (b) in a parton-model-like situation. These are for the case that the hadrons are close to back-to-back. The labels for the momentum classes of the subgraphs are: $H$ for hard, $S$ for soft, and $C_{(A)}$ and $C_{(B)}$ for collinear momenta.

The leading regions for the 2PI cross section in $e^{+} e^{-}$-annihilation are shown in Fig. 5. To avoid the complications associated with emission of extra partons at wide angle, I restrict attention to those regions that are leading when the measured hadrons are almost back-to-back; this is a situation appropriate for TMD factorization, with TMD ffs.

Figure 5(a) shows the general QCD case. Each subgraph is associated with a momentum class: collinear to one or other detected hadron, soft, or hard. The simplest characterization of the momentum classes is by the rough sizes, or scaling with $Q$, of momentum components in lightfront $[(+,-, T)]$ coordinates. For example, the canonical scalings of collinear momenta and soft momenta are:

$$
\text { Coll. A : }\left(Q, m^{2} / Q, m\right), \quad \mathrm{S}:(m, m, m) \text { or }\left(m^{2} / Q, m^{2} / Q, m^{2} / Q\right) \text {. }
$$

The alternative soft scalings are called soft and ultrasoft, for two different kinds of physics. Almost completely, the proofs in [6] are arranged to work uniformly for soft and ultrasoft. Although 
the scalings in (3.1) are very useful to gain intuition about the regions, it is important that all intermediate momenta between the canonical ones also contribute at leading power, and the proofs and the subtraction procedures must be arranged [6] to deal with this correctly.

Considerable complications in the proof arise because of the arbitrarily many gluons connecting the soft subgraph to the collinear subgraphs, and the collinear subgraphs to the hard subgraph. Only particular gluon polarizations contribute to leading power, and then certain kinds of Ward identities are used to obtain the factorized result, with gauge-invariant definitions of the factors.

If one had a theory without vector gluons, the leading regions would be of the much simpler form of Fig. 5(b). Here one already has a factorized form: a hard factor and two collinear factors. With appropriate approximations valid to leading power, the collinear factors become the TMD ffs in the natural TMD generalization of Eq. (2.2) for the back-to-back region.

Several issues can be identified as to whether a proof using order-by-order asymptotics in perturbation theory is sufficient:

- Perturbation theory is not literally convergent.

- Non-perturbative effects exist in QCD.

- Even if one ignores the non-convergence issue, the large- $Q$ asymptote and the sum of all orders of perturbation theory might not commute.

The last issue is rather important. As we will see, as $Q$ is increased, there is a set of graphs that are suggestive of the physics of string fragmentation. These are of order proportional to the available rapidity range, i.e., to $\ln \left(Q^{2} / \mathrm{m}^{2}\right)$, and they result in a filled-in rapidity gap. The asymptotics of each of these graphs is only reached when $Q$ is much larger than the value where the rapidity gap is filled.

\subsection{Non-perturbative-compatible structures in factorization proof}

For a general graph the leading-power analysis, following the Libby-Sterman [7, 8] argument, gives the structure in Fig. 5(a). It is natural to think of the blobs (or blocks) there, which are general subgraphs with particular momentum classes, as being (subtracted) matrix elements in full QCD, beyond perturbation theory. Furthermore, a space-time analysis shows that they essentially match a kind of (extended) parton-model view, in coordinate space. Approximations can be applied block-by-block rather than just individual-subgraph-by-individual-subgraph. In a case when no extra gluonic connections exist, we get Fig. 5(b), where the approximated collinear blobs directly correspond to ffs in the (TMD version of) factorization.

For these reasons, it would appear natural to expect that factorization has a broader generality than just order-by-order in perturbation theory.

The simplest parton-model-like regions, i.e., Fig. 5(b), lead to two separated jets, with a rapidity gap, of size approximately $\ln \left(Q^{2} / \mathrm{m}^{2}\right)$, between them. Moreover, in that situation, the jets have quark quantum numbers. Thus, it is wrong to take Fig. 5(b) at all literally in $\mathrm{QCD}$, since we know in reality large rapidity gaps are normally filled in.

Finally, we note that in the Drell-Yan process, there is a highly non-trivial cancellation of spectator-spectator interactions. The earliest proofs of cancellation, which essentially pre-date 
QCD, were fully non-perturbative [9, 10]; but these proofs used strict parton-model assumptions that are false in QCD. Collins, Soper and Sterman $[11,12]$ extended that work to full QCD, with an updated version in [6].

\section{What's missing?}

\subsection{Perturbative proof v. filled-in rapidity gap}

Suppose Fig. 5(b), were sufficient. Then we could interpret the blobs as full non-perturbative quantities. The natural leading-power approximations lead to factorization, complete with the natural operator definitions of the ffs. Even before the advent of QCD and the modern understanding of factorization, essentially these arguments were applied to relate the parton model for DIS to a full field theory of the strong interaction [13] (but with assumptions that are not valid in QCD).

We need something more general in QCD. Within the order-by-order analysis, we have Fig. 5(a). The approximations that lead to factorization require large rapidity differences between the subgraphs. For example, consider a soft gluon of momentum $k$ attaching to the collinear-to-A subgraph. With the canonical powers (3.1), the appropriate approximation for the product of collinear and soft subgraphs is made by

$$
A(k, \ldots)^{\mu} S(k, \ldots)_{\mu} \simeq A(\hat{k}, \ldots)^{+} S(k, \ldots)^{-}=A(\hat{k}, \ldots) \cdot \hat{k} \frac{1}{k^{-}} S(k, \ldots)^{-},
$$

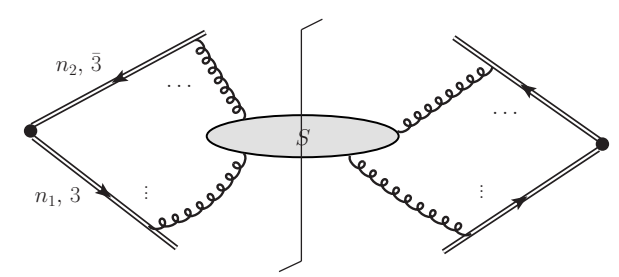

Figure 6: Generic soft factor.

where $\hat{k}=\left(0, k^{-}, 0_{\mathrm{T}}\right)$ in light-front $(+,-, T)$ coordinates, and we define collinear-to-A momenta to have large + components. The approximated graph has a factor of approximated gluon momentum contracted with the collinear subgraph with the same approximated momentum, i.e., $A(\hat{k}, \ldots) \cdot \hat{k}$. There exists a Ward identity to convert this soft-gluon attachment, and all the others in Fig. 5(a), to the form of a factorized soft piece that is a matrix element of Wilson lines, illustrated in Fig. 6. Essentially the same argument applies to the gluons connecting collinear to hard subgraphs, and results in the Wilson lines in the operator definition of the ffs.

A full treatment [6] needs to take account of subtractions and various kind of divergence; but the core of the argument consists of Eq. (4.1). Furthermore, in TMD factorization, it turns out to be useful to absorb $[6,14]$ square roots of the soft factor into the definitions of the collinear factors, i.e., the ffs, or more generally the pdfs and the ffs. Also, in collinear factorization a unitarity cancellation converts the soft factors into unity.

However, in reality the rapidity gaps are filled in, with about two particles per unit rapidity. So the assumption used in (4.1), i.e., of large rapidity differences, is not always true in real QCD; the assumption is appropriate only for the large- $Q$ asymptotics for an (arbitrary) individual graph.

\subsection{Soft-to-collinear approximation fails in string-compatible graphs}

We can even see the failure of the proof in a perturbative model that mimics the physics of string hadronization, as used in the PYTHIA event generator [15], or of a similar cluster hadronization used in HERWIG [16]. (See [1] for a review of hadronization and fragmentation.) 


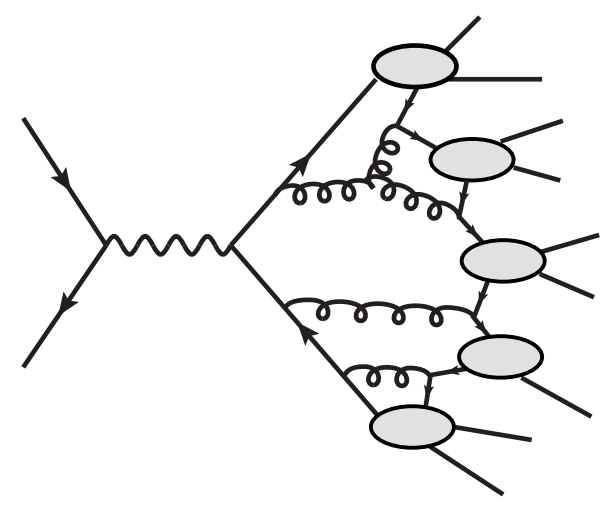

Figure 7: Cluster hadronization. The number of blobs for the final state interactions is proportional to $\ln \left(Q^{2} / m^{2}\right)$.

The model is illustrated in Fig. 7. It corresponds to the following semi-intuitive description: We have a quark and antiquark that are generated at a small distance and that propagate outward in opposite directions with energy approximately $Q / 2$, and with low virtuality. At early stages, gluons are emitted, and then quarkantiquark pairs are generated, which organize themselves into color-singlet hadrons. This is all qualitatively like the string model. Since virtualities decrease as we go away from the hard scattering, the relevant effective coupling increases (and ultimately we get to a non-perturbative region). Note that important contributions to the momenta of the central hadrons are from the soft gluons that are emitted early.

Let $\Delta y$ be total available rapidity range, about $\ln \left(Q^{2} / \mathrm{m}^{2}\right)$, and let $\delta y$ be typical cluster separation, i.e., $\Delta y / \#$ \#lusters. Experimentally $\delta y$ is typically small, a unit of rapidity or so.

Now suppose we were able apply soft-to-collinear approximations. Errors would now be a power of $e^{-\delta y}$, not $m / Q$, while the order of the relevant graphs increases with $\ln Q$. Although the middle blobs in Fig. 7 have soft momenta with momenta of order $(m, m, m)$, their structure fails to match Fig. 5(a) with its purely gluonic connections, and we do not have the rapidity differences needed in (4.1). In contrast, the soft-to-collinear approximation does apply to the gluons that are emitted early.

We thus see that the proof of factorization, as presented in the literature, does not work in graphical structures that are clearly important for getting the correct final state.

The above considerations do not themselves entail that factorization fails or even that the definitions of the pdfs and ffs are wrong, but only that there are some critical issues that are not fully understood. As already pointed out, the space-like separation between the dominant hadronization processes in different jets strongly suggests that some kind of factorization is valid, with something like ffs. But the coordinate-space description does not itself allow to obtain operator definitions of the ffs in a simple way. Furthermore, such coordinate-space arguments are quite foreign to the momentum-space methods used in factorization proofs.

A more indirect approach might be fruitful. The coordinate-space factorization argument could be applied to the operator matrix elements in the definition of ffs, since these involve color flows in very different directions. From this approach one may well recover the usual factorization results, but now demonstrated to be valid in full QCD. But completing such a program is for the future.

\section{Discussion}

The overall issue addressed in this paper is: How well do we know that factorization is valid? Then, given that we do find that the derivations need extensions, at least concerning hadronization, a question for the future is to ask what new phenomena and properties could be found from a better understanding. 
We saw that string-type hadronization [1] does not match perturbative derivations of factorization, even in a perturbative model. This specific problem applies everywhere that cross sections are differential in final-state particles. This is only one part of the overall issue of asking how to establish factorization theoretically within full QCD as opposed to literally order-by-order in perturbation theory. Even so, as I pointed out in Sec. 3.2, there are many parts of the current proofs that do transcend a purely perturbative treatment.

The arguments in this paper do not themselves imply that factorization is falsified. The opposite ends of a Lund string are at space-like separation, so we can still expect independent hadronization (except for the effects of QM entanglement). There is tension or even a clash, however, between the coordinate-space picture that gives this expectation, and the momentum-space formulation of most work on factorization.

There are a number of directions for future work that seem useful:

- Do the issues I raised actually impact factorization formulas with fragmentation functions? This applies to both collinear and TMD factorization.

- We need to find an improved formulation, perhaps involving a proper systematic interface between standard pQCD constructs and string-like constructs.

- What other similar issues affect derivations of factorization, including those with pdfs?

- We need improved and quantitative methods for analyzing graphs and amplitudes in coordinate space, and for relating them to work in momentum space.

- What other implications are there?

- What other problems are there?

- How can one understand better the relation with the methods of soft-collinear effective theory?

A better formulation should assist in better matching the algorithms used in Monte-Carlo event generators to the TMD factorization formalism.

\section{References}

[1] B. R. Webber, Fragmentation and hadronization, Int. J. Mod. Phys. A15 (2000) 577-606, [hep-ph/9912292].

[2] X. Artru and G. Mennessier, String model and multiproduction, Nucl. Phys. B70 (1974) 93-115.

[3] B. Andersson, G. Gustafson, G. Ingelman and T. Sjostrand, Parton fragmentation and string dynamics, Phys. Rept. 97 (1983) 31-145.

[4] X. Artru, Classical string phenomenology. How strings work, Phys. Rept. 97 (1983) 147-171.

[5] B. Andersson, The Lund Model. Cambridge University Press, Cambridge, 1998, 10.1017/CBO9780511524363.

[6] J. C. Collins, Foundations of Perturbative QCD. Cambridge University Press, Cambridge, 2011, 10.1017/CBO9780511975592. 
[7] S. B. Libby and G. Sterman, Mass divergences in two-particle inelastic scattering, Phys. Rev. D18 (1978) 4737-4745.

[8] S. B. Libby and G. Sterman, Jet and lepton-pair production in high-energy lepton-hadron and hadron-hadron scattering, Phys. Rev. D18 (1978) 3252-3268.

[9] C. E. DeTar, S. D. Ellis and P. V. Landshoff, Final state interactions in large transverse momentum lepton and hadron production, Nucl. Phys. B87 (1975) 176-188.

[10] J. L. Cardy and G. A. Winbow, The absence of final state interaction corrections to the Drell-Yan formula for massive lepton pair production, Phys. Lett. B52 (1974) 95-96.

[11] J. C. Collins, D. E. Soper and G. Sterman, Factorization for short distance hadron-hadron scattering, Nucl. Phys. B261 (1985) 104-142.

[12] J. C. Collins, D. E. Soper and G. Sterman, Soft gluons and factorization, Nucl. Phys. B308 (1988) 833-856.

[13] P. V. Landshoff and J. C. Polkinghorne, Models for hadronic and leptonic processes at high-energy, Phys. Rept. 5 (1972) 1-53.

[14] M. G. Echevarría, A. Idilbi and I. Scimemi, Factorization theorem for Drell-Yan at low $q_{T}$ and transverse momentum distributions on-the-light-cone, JHEP 1207 (2012) 002, [1111.4996].

[15] T. Sjöstrand, S. Mrenna and P. Z. Skands, PYTHIA 6.4 physics and manual, JHEP 05 (2006) 026, [hep-ph/0603175].

[16] G. Corcella et al., HERWIG 6.5: an event generator for hadron emission reactions with interfering gluons (including supersymmetric processes), JHEP 01 (2001) 010, [hep-ph/0011363]. 